\title{
Evaluation of the Blood-Glucose Reducing Effects of Aqueous Extracts of the Selected Umbelliferous Fruits Used in Culinary Practices
}

\author{
K Sushruta $^{1}$, S Satyanarayana ${ }^{2}$, N Srinivas ${ }^{2}$., J Raja Sekhar ${ }^{2}$ \\ 1.Elrazi College of Medical and Health Sciences, Khartoum, Sudan. \\ 2.University College of Pharmaceutical Sciences, Andhra University, Visakhapatnam, A.P., India - 530003.
}

\begin{abstract}
Purpose: Oxidative stress in diabetes coexists with a reduction in the antioxidant status, which can increase the deleterious effects of free radicals. Many plant extracts and plant products have been shown to have significant antioxidant activity. The role of antioxidant plants in diabetes has not been studied. Food additives like spices, which have free radical scavenging activity, may also be useful in maintaining normal glucose levels in diabetic patients. The objective of the study is to evaluate the aqueous extracts of selected umbelliferous fruits for hypoglycaemic and antidiabetic activities in normal and diabetic rats respectively.

Methods: All the aqueous extracts of (Caraway) CA, (Coriander) CO, (Cumin) CU, (Dill) DI and (Fennel) $F E$ were administered at dose levels of $300 \mathrm{mg} / \mathrm{kg}$ body weight orally to different groups each containing 5 animals. A control group was also maintained simultaneously and received distilled water orally. Blood samples were collected from retro-orbital plexus of each rat before and at 2, 4, 6 and $8 \mathrm{~h}$ after extract administration and were analyzed for glucose content by using glucose oxidase peroxidase (GOD-POD) method using a visible spectrophotometer at $505 \mathrm{~nm}$.

Results: In normal rats only caraway extract produced significant hypoglycaemic action. The rest of the extracts produced hypoglycaemia, which was not significant different from that of control animals. In diabetic rats also the caraway was found to be the best among the extracts.

Conclusion: The study indicates that since the umbelliferous fruits are used in the preparation of foods, they may be useful in the control of postprandial rise of blood glucose particularly in diabetic condition. Additionally, their daily use may help in reducing complications associated with chronic diabetes.
\end{abstract}

Key words: Diabetes, Oxidative stress, Glucose Oxidase - Peroxidase (GOD-POD), Antioxidants. 


\section{INTRODUCTION}

Diabetes mellitus is the common metabolic disorder charecterised by hyperglycaemia. There are an estimated 143 million people worldwide suffering from the disease ${ }^{1}$ and this is almost five times the estimate ten years ago. It has been predicted that the number may probably double by the year $2030^{2}$. Therefore, the human population worldwide appears to be in the midst of an epidemic of diabetes. The introduction of Insulin and later oral hypoglycaemic agents, revolutionized the management of diabetes mellitus In spite of advances in drug management of diabetes, there are still complications and adverse drug reactions. None of them were unequivocally successful in maintaining normal blood glucose levels and in avoiding complications.

In spite of all the advances in therapeutics, diabetes still remains a major cause of morbidity and mortality in the world. Aside from drugs, diet control also plays a key role for the management of diabetes. Foods of high fiber content like fruits, vegetables, grains, beans and foods that contain less amount of fat, cholesterol, sugar and salt are useful in maintaining normal glucose levels in diabetic patients.

Free radicals have been implicated in the causation of several disorders, which includes diabetes, and the agents that scavenge free radicals may have great potential in ameliorating these disease processes ${ }^{3}$. Antioxidants play an important role in protecting the human body against damage by reactive oxygen species ${ }^{4}$. Increased oxidative stress has been postulated in the diabetic state ${ }^{5}$. Oxidative stress in diabetes coexists with a reduction in the antioxidant status ${ }^{6}$, which can increase the deleterious effects of free radicals.

It has also been shown that alloxan induces its diabetogenic activity mainly by inducing the formation oxygen free radicals and thereby damaging the pancreas 7 . Supplementation with antioxidants may have a chemoprotective role in the diabetes ${ }^{8}$. Present therapeutic strategies mostly try to relieve the clinical manifestation of diabetes and complications. Since diabetes seems to be a stress - related disorder, antioxidants may be useful.

Many plant extracts and plant products have been shown to have significant antioxidant activity ${ }^{9-11}$ but the role of antioxidant plant products in diabetes has not been investigated 12. Thus food additives like spices, which have free radical scavenging activity, may be useful in controlling glucose levels in diabetic patients.

The objective of the study was to evaluate the selected umbelliferous fruit aqueous extracts for hypoglycaemic and antidiabetic activities in normal and diabetic rats respectively.

\section{MATERIALS AND METHODS \\ Studies in normal rats}

Animals-Albino rats (Wistar strain) of either sex weighing 200-300 g procured from Chakraborty Enterprises, Kolkata were used in the study. Animals were divided into six groups each containing five and were provided with standard Gold Mohar Pellet diet and water adlibitum. They were fasted for about $18 \mathrm{~h}$ prior to the experiment, with access to water. During the experiment, water was removed while fasting was continued. The experimental protocol was approved by the Institutional Animal Ethics Committee and by the animal regulatory body of the Indian Government (Reg. No.516/01/A/CPCSEA).

All the aqueous extracts of (Caraway) $\mathrm{CA}$, (Coriander) CO, (Cumin) CU, (Dill) DI and (Fennel) FE were administered at dose levels of $300 \mathrm{mg} / \mathrm{kg}$ body weight orally (using orogastric tube) ${ }^{13}$ to different groups each containing 5 animals. A control group was maintained simultaneously and received distilled water orally.

Blood samples were collected from retro-orbital plexus of each rat before and at 2, 4, 6 and $8 \mathrm{~h}$ after extract administration and were analyzed for glucose content by using glucose oxidase peroxidase (GOD-POD) method using a visible spectrophotometer at $505 \mathrm{~nm}$. 
Table 1: Mean percentage serum glucose reduction with control and selected umbelliferous fruit aqueous extracts in normal rats $(n=5)$

\begin{tabular}{ccccccc}
\hline Time (h) & Control & Caraway & Coriander & Cumin & Dill & Fennel \\
\hline 0 & 0 & 0 & 0 & 0 & 0 & 0 \\
2 & $-2.03 \pm 0.6$ & $6.09 \pm 3.03^{*}$ & $2.61 \pm 0.78$ & $-0.99 \pm 0.80$ & $0.55 \pm 0.89$ & $0.01 \pm 1.36$ \\
4 & $0.718 \pm 1.24$ & $14.51 \pm 1.55^{*}$ & $5.54 \pm 0.59^{*}$ & $1.18 \pm 0.80$ & $4.41 \pm 1.21$ & $5.02 \pm 1.80$ \\
6 & $5.25 \pm 1.17$ & $22.58 \pm 1.97^{*}$ & $6.50 \pm 0.59$ & $9.46 \pm 0.60^{*}$ & $7.73 \pm 1.18$ & $7.04 \pm 1.42$ \\
8 & $0.77 \pm 0.52$ & $2.99 \pm 1.04^{*}$ & $3.75 \pm 0.43$ & $2.69 \pm 0.35$ & $1.80 \pm 0.53$ & $1.76 \pm 0.33$
\end{tabular}

Values are Means \pm S.E.M; $n=$ number of animals; ${ }^{*} p<0.05$

\section{Preparation of the extract solution}

The extracts of CA, CO, CU, DI and FE being aqueous, all the solutions were prepared by dissolving the extracts in distilled water.

\section{Studies in diabetic rats: Induction of diabetes}

The same groups used earlier (normal) were used to induce diabetes after a washout period of 10 days. These animals were injected with freshly prepared aqueous solution of alloxan monohydrate (Sigma chemical company, USA) at a dose of $100 \mathrm{mg} / \mathrm{kg}$ body weight by intraperitoneal route. $10 \%$ dextrose was there after administered orally to combat the immediate hypoglycaemia that could occur. The serum glucose was observed $20 \mathrm{~h}$ after alloxanisation. The serum glucose levels were observed daily for 5 days. The rats showed serum glucose level ranging $283-343 \mathrm{mg} / \mathrm{dL}$. Animals were fed with the same standard diet and water ad libitum. They were fasted for about $18 \mathrm{~h}$ prior to the experiment providing access to water.

All the aqueous extracts of $\mathrm{CA}, \mathrm{CO}, \mathrm{CU}$, $\mathrm{DI}$ and FE were administered at dose level of $300 \mathrm{mg} / \mathrm{kg}$ body weight orally to their respective groups each containing 5 rats. The control group received distilled water only. Blood samples were collected and analyzed as mentioned earlier.

\section{Data and statistical analysis}

Data were expressed as Mean \pm Standard Error of Mean (SEM). The significance of blood glucose reduction produced by the aqueous extracts compared control was determined by applying student's unpaired ' $\mathrm{t}$ ' test. $P$ values of $<0.05$ were considered to be statistically significant.

\section{RESULTS AND DISCUSSION}

The comparative results of \% serum glucose reduction with aqueous extracts in normal rats were given in Table 1 . The comparative results of $\%$ serum glucose reduction with alloxan induced diabetic rats were given in Table 2. Evidence has been accumulated in the past few years supporting that diabetes was precipitated by stress ${ }^{5,14}$. Additionally, it was also reported that hyperglycaemia itself increases stress ${ }^{15}$. So it was clear that antioxidants can prevent precipitation of diabetes and also control hyperglycaemia. Since the umbelliferous fruit aqueous extracts exhibited antioxidant activity in in vitro and in vivo studies ${ }^{16}$, they were evaluated for their hypoglycaemic and antihyperglycaemic activities in normal and alloxan induced diabetic rats. The diabetes was induced with alloxan, since it was more economical and easily 
Sushruta et al

Table 2: Mean percentage serum glucose reduction with control and selected umbelliferous fruit aqueous extracts in alloxan induced diabetic rats $(n=5)$

\begin{tabular}{ccccccc}
\hline Time (h) & Control & Caraway & Coriander & Cumin & Dill & Fennel \\
\hline 0 & 0 & 0 & 0 & 0 & 0 & 0 \\
2 & $0.51 \pm 2.83$ & $0.97 \pm 0.60$ & $4.83 \pm 2.06$ & $3.43 \pm 0.79$ & $4.74 \pm 2.82$ & $3.18 \pm 3.60$ \\
4 & $3.09 \pm 2.67$ & $8.54 \pm 0.80^{\star}$ & $12.12 \pm 2.10^{\star}$ & $12.36 \pm 0.78^{\star}$ & $16.16 \pm 2.60^{\star}$ & $5.16 \pm 3.88$ \\
6 & $0.57 \pm 2.03$ & $28.95 \pm 0.09^{\star}$ & $19.62 \pm 1982^{\star}$ & $19.91 \pm 0.21^{\star}$ & $21.4 \pm 2.66^{\star}$ & $15.42 \pm 2.56^{\star}$ \\
8 & $3.90 \pm 1.23$ & $23.73 \pm 0.94^{\star}$ & $12.84 \pm 3.64^{\star}$ & $15.38 \pm 0.58^{\star}$ & $14.35 \pm 3.79^{\star}$ & $12.15 \pm 2.88^{\star}$ \\
\hline
\end{tabular}

Values are Means \pm S.E.M; $n=$ number of animals; ${ }^{*} p<0.05$

available. Moreover, alloxan was reported to produce diabetes by damaging pancreas by free radical related mechanisms ${ }^{17}$.

Rat was used since it was routinely used animal model for quick screening of drugs for their hypoglycaemic/antihyperglycaemic action. Since small amount of blood was required for glucose analysis, the blood samples were collected by retro-orbital puncture as it was reported to be good method when small samples of blood were required ${ }^{18}$.

In normal rats only caraway extract produced appreciable hypoglycaemic action. The rest of the extracts produced hypoglycaemia, which was not very different from that of control animals. It was also reported that caraway extract have highest activity in scavenging superoxide radicals and lipid peroxidation inhibition. So it appears that superoxide radical scavenging action and inhibition of lipid peroxidation were responsible for the observed hypoglycemic action in normal rats. Recent report indicated that 14 days daily treatment with alcoholic extract of caraway (20 mg/kg body weight) produced normalization of blood glucose in streptozotocin induced diabetic rats while it had no effect on blood glucose of normal rats ${ }^{18}$. However in the present study aqueous extract of caraway produced hypoglycaemia in normal rats. The difference may be attributed to the difference in the active ingredients present in the extract since one was aqueous and the other was alcoholic or it may be due to difference in the rat strain.

With alloxan treatment the blood glucose was raised and it was in the range of 283-343 $\mathrm{mg} / \mathrm{dL}$ in different rats after stabilization for a period of 5 days. Single dose (300 mg/kg body weight, oral) treatment with the extracts in different groups produced antihyperglycaemic effect (antidiabetic effect). In diabetes also the caraway was found to be the best among the extracts with peak effect at $6 \mathrm{~h}$ with $28.95 \%$ reduction in blood glucose level. The peak effects of cumin, dill and fennel were $19.91 \%, 21.47 \%$, and $15.42 \%$ respectively a $6 \mathrm{~h}$. The peak effect of coriander was $19.62 \%$ at $6 \mathrm{~h}$. The common feature of all these extracts was that they produced a peak effect at $6 \mathrm{~h}$ only. All of them produced better reduction in blood glucose in diabetic condition compared to nondiabetic condition indicating that antioxidants were more useful in stress (diabetes) than in normal condition.

In the present study also the extract of caraway, cumin and others contained flavonoids ${ }^{20-22}$ and the same might have been responsible for the observed activity. The present study showed that reduction of blood glucose was more in diabetic rats compared to non-diabetic rats at the same dose level of the extracts. Since stress (free radical generation) was 
responsible for alloxan-induced diabetes ${ }^{5}$ and high glucose level was reported to produce increased stress ${ }^{15}$, it was likely that the antioxidant activity of the extracts produced better response in such stressful conditions compared to normal (non-diabetic) condition.

Finally it can be concluded that Caraway was found to be best among the extracts evaluated for glucose lowering activity. Since the umbelliferous fruit is used in the preparation of curries etc particularly in Indian/Chinese foods they are likely to be useful to control postprandial rise of blood glucose particularly in diabetic condition. Additionally, their daily use may help in reducing complications associated with chronic diabetes.

\section{Acknowledgements:}

The financial support by the CSIR, New Delhi to Mr. K. Sushruta for pursuing Ph.D. programme is gratefully acknowledged. The authors are indebted to Sri G.Gangaraju and the scientists of Laila impex, Vijayawada for supplying the aqueous extracts.

\section{References}

1. Harris SB, Macaulay AC. Diabetes management: new evidence-based recommendations. Highlights of the 1998 Canadian clinical practice guidelines. Canadian Diabetes Association.Can Fam Physician. 1998; 44: 2465-6

2. Kingh $H$, Aubert RE, Herman WH. Global burden of diabetes, 1995-2025: prevalence, numerical estimates, and projections. Diabetes Care. 1998 Sep; 21(9): 1414-31.

3. Wilson RL. Free Radicals and Tissue Damage, Mechanistic Evidence from Radiation Studies. In: Biochemical Mechanisms of Liver Injury. Academic Press, New York. 1988; pp.123.

4. Lollinger J. Free radicals and Food additives. Ed. by Taylor and Francis, London, 1981; p: 121.

5. Lawrence JC, Jill SG, Eric $P D$, Joyle $A D$, Donald $D L$, Mark AY. Effect of antioxidant treatment on streptozotocin induced diabetic rats on endoneurial blood flow, motor nerve conduction velocity and vascular reactivety of epineural arterioles of the sciatic nerve. Diabetes 2001; 50: 1927-1937.

6. Collier A, Wilson R, Bradley $H$, Thomson JA, Small M. Free radical activity in Type II Diabetes. Diabetic. Med. 1990; 7: 27-30.

7. Halliwell B, Gutteridge JMC, Free Radicals in Biology and Medicine. ( $1^{\text {st }}$ ed.), Clarendon Press. Oxford, 1985; $279-313$.
8. Logani MK, Davis RE. Lipid peroxidation in biologic effects and antioxidants: a review. Lipids. 1979; 15: 485-493.

9. Anjali $P$ and Manoj KM: Some comments on Diabetes and herbal therapy. Ancient. Sci. life. 1995; 15: 27 29.

10. Jain SR and Sharma SN. Hypoglycaemic drugs of Indian indigenous origin. Planta Medica, 1967; 15: 439-442.

11. Nagarajan S, Jain HC, and Aulakh GS. Indigenous plants used in the control of diabetes. Publication and Information Directorate , CSIR, New Delhi;1987; p: 586.

12. Sabu MC and Kuttan R: Antidiabetic activity of medicinal plants and its relationship with their antioxidant property. J. Ethnopharmcolgy. 2002; 81: $155-160$

13. Ghosh MN. Fundamentals of experimental Pharmacology $2^{\text {nd }}$ edition. Scientific book agency. Kolkata. 1984; p: 89.

14. Oberly LW: Free radicals and diabetes. Free. Radic. Biol. Med. 1988; 5: $113-124$

15. West IC: Radicals and oxidative stress in diabetes. Diabet. Med. 2000; 17: 171-180.

16. Satyanarayana S, Sushruta K, Sharma GS, Srinivas. N Subba Raju GV Antioxidant activity of the aqueous extracts of spicy food additives - evaluation and comparison with ascorbic acid in in-vitro systems. J Herb Pharmacother 2004; (2):1-10

17. Heikkila RE. The prevention of alloxan-induced diabetes in mice by dimethyl sulfoxide. Eur. J. Pharmacol. 1977; 44(2): 191-193.

18. Riley V. Adaptation of orbital bleeding technique to rapid serial blood studies. Proc. Soc. Exp. Biol. Med. 1960; 104:751-754.

19. Eddouks M, Lemhadri A, Michel JB. Caraway and Caper: potential anti-hyperglycaemic plants in diabetic rats. J.Ethnopharmacol. 2004; 94(1): 143148

20. Kallio $H$, Kerrola $K$, Alhonmaki $P$. Carvone and limonene in caraway fruits (carum carvi l.) Analyzed by supercritical carbon dioxide extraction - gas Chromatography. J. Agr. Food. Chem. 1994; 42 (11): 2478-2485

21. Harborne JB, Williams CA, Comparative biochemistry of the flavonoids. Xvii. Flavonoid patterns in the fruits of the umbelliferae. Phytochemistry. 1972; 11 : $1741-1750$

22. Akunzemann J, Herrmann K. Isolation and identification of Flavon (ol)-o-glycosides in caraway (carum carvi I.) fennel (foeniculum vulgare mill.), anise (pimpinella anisum I.), and coriander (coriandrum sativum I.), and of flavone-c-glycosides in anise. I. Phenolics of spices. Z. Lebensm-Unters forsch. 1977; 164: 194-200. 